\title{
EFECTO DEL SITIO Y DE DIFERENTES ASOCIACIONES ARBÓREAS EN EL CRECIMIENTO Y CALIDAD FUSTAL DEL ROBLE EUROPEO (Quercus robur L.) EN EL SUR DE CHILE
}

\author{
Loewe, Verónica ${ }^{5}$; Siebert, Herbert ${ }^{6}$ y Herrera, Rodrigo ${ }^{7}$
}

\section{RESUMEN}

El proceso de diversificación forestal en Chile ha estimulado el interés por cultivos y modelos productivos no tradicionales, dentro de los cuales las plantaciones mixtas han mostrado utilidad para la realidad forestal al favorecer el crecimiento en altura y diámetro. Debido a la ductilidad de su madera, el roble europeo (Quercus robur L.) es altamente demandado en el mercado internacional. Este estudio determinó el efecto en el crecimiento y la calidad fustal del $Q$. robur de varias asociaciones establecidas y manejadas mediante silvicultura con apego a la naturaleza en el fundo Miraflores (Lanco, Región de los Ríos, Chile), haciendo un muestreo de 200 árboles futuro por asociación (una pura y nueve mezclas diferentes). Los resultados indican que la proporción de árboles futuro es mayor en las asociaciones mixtas respecto a la pura; y que el crecimiento en altura y diámetro en la situación pura es mayor que en las mezclas analizadas. No obstante lo anterior, no se recomienda la plantación pura, pues este manejo provoca una importante penetración de luz, que favorece el desarrollo de características que descalifican la calidad de madera, como la aparición de brotes epicórmicos. Se concluyó que las asociaciones más favorables para el desarrollo de Quercus robur incluyen arce (Acer pseudoplatanus) y castaño (Castanea sativa), seguida de pino oregón (Pseudotsuga mensiezii) y castaño.

Palabras Clave: Diversificación, Plantaciones mixtas, Encino, Silvicultura con apego a la Naturaleza, Producción de madera de alto valor, Monocultivo.

\section{SUMMARY}

The forest diversification process in Chile has stimulated the interest for cultural and productive non-traditional models, among which the mixed plantations have shown usefulness for the forest activity, since in many cases they favor height and diameter (DBH) growth. Due to the ductility of its wood, Quercus robur is highly demanded on international markets. This study determined the effect in the growth and trunk quality of several associations with $Q$. robur established and managed using silviculture mimicking nature in the property Miraflores (Lanco, Region de Los Ríos, Chile); by sampling 200 future high quality trees per association (1 pure and 9 mixtures). Results showed a higher proportion of future trees in the mixed plantations. Height and diameter growth in the pure situation were higher than in the different mixtures, but the proportion of superior future trees was higher in the later. Consequently, it is not recommended pure plantations given the significant penetration of light that favors the development of characteristics that disqualify wood quality. It is concluded that mixed plantations are more favorable for the development of $Q$. robur, species that grows better when associated to Maple (Acer pseudoplatanus) and Chestnut tree (Castanea sativa), followed by the association with Douglas Fir (Pseudotsuga menziesii) and Chestnut tree.

Keywords: Diversification, Mixed plantations, Oak, Quercus robur, Forestry mimicking nature, Highvalue timber production, Monoculture.

\footnotetext{
${ }^{5}$ Instituto Forestal (INFOR), Sucre 2397, Santiago.

vloewe@infor.cl

${ }^{6}$ Instituto de Silvicultura, Universidad Austral de Chile. hsiebertw@surnet.cl

${ }^{7}$ Departamento de Fiscalización, CONAF - Oficina Central. rodrigo.herrera@conaf.cl
} 


\section{INTRODUCCIÓN}

Quercus robur L., conocido en Chile como roble europeo o encino, posee una madera de alto valor apreciada en el mercado mundial por su durabilidad, buenas propiedades tecnológicas y versatilidad, que permiten diferentes usos, desde construcciones hidráulicas y navales, edilicias, muebles, hasta barricas de vino y leña (Mori et al., 2007). En Chile, aun cuando se considera como una especie promisoria (Loewe, 1998), sus potencialidades no han sido explotadas, principalmente por el desconocimiento de la especie por parte de silvicultores y de la industria.

Es una especie nativa de Europa, donde se distribuye desde los $60^{\circ}$ de latitud norte, en el mediterráneo se encuentra en los Apeninos y en Grecia, principalmente en valles (Suszka et al., 1994). Crece en suelos profundos, frescos, generalmente ácidos y en zonas con un período seco nulo o muy corto, desde el nivel del mar hasta $1.000 \mathrm{~m}$ de altitud (Menéndez, 2006). Al principio se ve favorecida por un acompañamiento lateral, pero conforme pasan los años se hace más exigente en espacio vital y requiere plena luz (Álvarez et al., 2000), lo que le confiere el carácter de semitolerante (tolerante en su juventud e intolerante en la adultez) (Loewe, 2009). De hecho, Savill y Evans cit. por Aguayo (2000) reportan que el máximo crecimiento en altura del primer año del brinzal ocurre en condiciones cercanas al $30 \%$ de luz, pero que en años posteriores necesita de al menos un $50 \%$. Este fototropismo positivo provoca torceduras que induce a malformaciones de los individuos, y favorece la aparición de brotes epicórmicos (Burschel y Huss, 1997), especialmente cuando hay un incremento repentino de luz como respuesta a podas o raleos (Wignal et al. cit. por Aguayo, 2000).

Se desarrolla bien sobre sustratos tanto ácidos como calizos, pero se desarrolla mejor en suelos ácidos, arcillo-ácidos, arcillo-arenosos o arcillosos medianamente compactos (Najera y Ángulo y López, 1969). Según Joyce et al. (1998) los suelos óptimos son de textura fina, ácidos, profundos y fértiles.

Habita zonas con clima templado-cálido a templado-frío, desde semi-secos a muy húmedos (Najera y Angulo, y López, 1969). Requiere climas de tendencia atlántica con un mínimo de humedad (precipitaciones anuales de al menos $600 \mathrm{~mm}$ ), no tolerando sequías estivales fuertes (Álvarez et al., 2000); es afectada por heladas tardías, ya que temperaturas de - $3^{\circ} \mathrm{C}$ matan los brotes nuevos, por lo que es una de las últimas especies en florecer (Savill, 1991). Es una especie pionera debido a su habilidad para sobrevivir con muchos competidores una vez establecida, para crecer en una amplia gama de suelos, y de buena dispersión de semillas, lo que permite su difusión (Savill, 1991).

En Europa la actividad antrópica redujo fuertemente la distribución del roble europeo (Ducousso y Bordacs, 2004), ya que los árboles con buenas características madereras se cortaron intensamente (Loewe, 2002), generando una degradación genética importante (Álvarez et al., 2000). Debido a ello, se ha probado un sistema de plantación de 2 plantas de la misma especie separadas por 30-50 cm, para elegir la planta mejor adaptada al sitio luego de haber probado in situ su desarrollo y hábito (Buresti y Mori 1999, citado por Loewe, 2003), sistema conocido como doble planta.

En Chile esta especie se ha usado en áreas verdes, parques y jardines, como especie ornamental, para enriquecer bosque nativo y estabilizar laderas. No obstante, no existe mayor conocimiento sobre el cultivo de la especie, salvo casos aislados, como el del Fundo Miraflores.

Las plantaciones mixtas, que pueden ser realizadas tanto bajo un modelo de silvicultura tradicional como también bajo un sistema de arboricultura de calidad, corresponden a modelos que asocian especies principales que generan productos de alto valor al final de la rotación, con especies acompañantes que tienen como función favorecer el crecimiento de la especie principal, mejorando además su forma, lo que conduce a una mejora en la calidad de los productos (Loewe y González, 2001). Adicionalmente, la(s) especie(s) principal(es) genera(n) producto(s) de alto valor al momento de la cosecha (aserrable y foliable), y la(s) especie(s) acompañante(s) pueden también 
aportar productos intermedios como postes, polines, frutos $u$ otros, a obtener en este mismo transcurso de tiempo (Loewe, 2000).

Si son bien diseñadas, las plantaciones mixtas pueden diversificar la producción, reducir los riesgos fitosanitarios, facilitar algunas prácticas de cultivo (poda y control de malezas), mejorar el paisaje (Gabriel et al., 2005; Hung et al., 2011) y elevar la calidad de la madera al reducir tanto las bifurcaciones como la cantidad de ramas gruesas, que la degradan (Hung et al., 2011). La asociación con especies fijadoras de nitrógeno presenta mayores crecimientos en relación a plantaciones puras (Forrester et al., 2011; Hung et al., 2011).

En Latinoamérica, la literatura sobre plantaciones mixtas es escasa, con algunas investigaciones reportadas por Piotto et al. (2004) en Costa Rica, y por Loewe y González (2006) y Loewe et al. (2013) en Chile.

El roble europeo en plantaciones mixtas generalmente es dominante o codominante, competencia que en plantaciones puras se ve incrementada debido a que todos los integrantes del rodal buscan dominar. Buresti et al. (1998) indican que la plantación pura no es favorable para la especie, dado que al ralear penetra mayor cantidad de luz, y el rodal reacciona elevando el crecimiento, el grosor de las ramas, y también emitiendo numerosos brotes epicórmicos, lo que deteriora la calidad de la madera por la pérdida de homogeneidad de los anillos de crecimiento y por la mayor cantidad de nudos y marcas, dificultándose el acceso a los exigentes mercados de esta madera.

Considerando la importancia económica de la especie y el interés por alternativas productivas en Chile, se evaluó el efecto de distintas asociaciones de especies arbóreas con $Q$. robur con relación a: i) calidad fustal como proporción de árboles maderables que llegarán a la madurez (denominados $M$ ); ii) crecimiento en diámetro y altura; iii) presencia de defectos de disminuyen la calidad de la madera.

\section{MATERIAL Y MÉTODO}

\section{Área de Estudio}

Las plantaciones evaluadas se ubican en el Fundo Miraflores, comuna de Lanco, en la precordillera andina de la provincia de Valdivia, Región de los Lagos (Latitud 39³0'S y Longitud 72ํㅜㅇ'W) (González, 2004), que se encuentra en suelos clase VI-VII de la serie Correltúe (trumaos), de origen volcánico.

Predomina una topografía de lomajes suaves con substrato semicompacto, frecuentemente cementado, recubierto por materiales finos de textura franco limosa a franco arenosa fina, de profundidad variable y de óptimas condiciones físicas, con alto contenido de materia orgánica (Rodríguez, 1990).

Presenta altitudes entre 100 y $700 \mathrm{msnm}$, clima templado lluvioso, con más de $2.000 \mathrm{~mm}$ de precipitación anual repartidos en el año; sobre los $500 \mathrm{msnm}$ se presenta nieve ocasional en invierno. Las temperaturas oscilan entre $-3^{\circ} \mathrm{C}$ de mínima y $35^{\circ} \mathrm{C}$ de máxima, con una temperatura media de $12^{\circ} \mathrm{C}$ y diez días de heladas al año (Siebert et al., 2003).

\section{Diseño}

Las plantaciones tienen una densidad de 1.634 plantas/ha, son coetáneas y con diseños espaciales similares, variando las especies acompañantes (Figura $\mathrm{N}^{\circ} 1$ y Cuadro $\mathrm{N}^{\circ} 1$ ). El estudio cubrió una superficie de 30 ha con distinta exposición (laderas sombrías o soleadas). La terminología de ladera sombría incluye pendientes expuestas al Sur, Sureste y Este, mientras que las laderas asoleadas son las que miran hacia el Norte, Noreste y Oeste. 
Este concepto, según Weinstroer et al. (2003) se basa en las particularidades meteorológicas de Chile alrededor de los $40^{\circ}$ de Latitud Sur, donde el promedio de horas con sol es más corto en la mañana, con la posición Este del sol, y más largo en la tarde, con la posición Oeste del sol. Este efecto es más pronunciado en la precordillera y cordillera que en el valle central, acentuado por la sombra orográfica de la Cordillera de los Andes al levantarse el sol.

El objetivo de estas plantaciones es producir madera de elevada calidad (troncos únicos, rectos, cilíndricos, con crecimiento regular y sin defectos) (Berti et al., 2003).

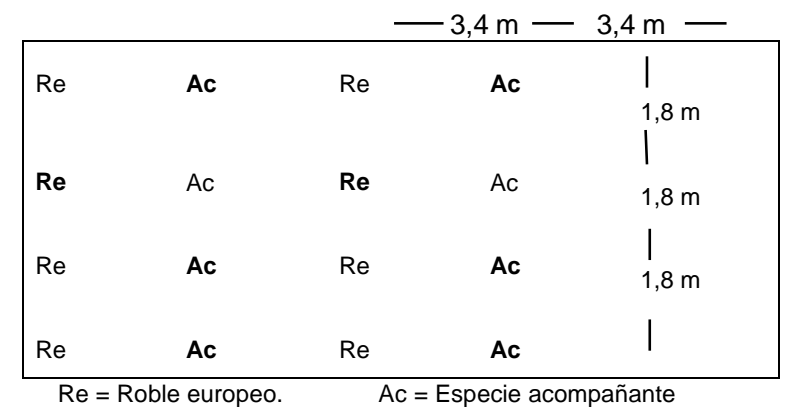

Figura $\mathbf{N}^{\circ} 1$

ESQUEMA DE PLANTACIÓN MIXTA EN HILERAS

\section{Cuadro $N^{\circ} 1$
IDENTIFICACIÓN Y COMPOSICIÓN DE RODALES ESTUDIADOS}

\begin{tabular}{|c|c|c|c|}
\hline $\begin{array}{c}\text { Rodal } \\
\text { № }\end{array}$ & Composición & Ladera & $\begin{array}{c}\text { Edad } \\
\text { (años) }\end{array}$ \\
\hline 1 & Quercus robur- Quercus robur & Luz & 13 \\
\hline 2 & Quercus robur - Castanea sativa & Sombra & 19 \\
\hline 3 & Quercus robur - Acer pseudoplatanus - Castanea sativa & Luz & 19 \\
\hline 4 & Quercus robur - Acer pseudoplatanus - Castanea sativa & Luz & 19 \\
\hline 5 & Quercus robur - Pseudotsuga menziesii - Castanea sativa & Luz & 19 \\
\hline 6 & Quercus robur - Pseudotsuga menziesii - Castanea sativa & Sombra & 20 \\
\hline 7 & Quercus robur - Cupressus macrocarpa-Castanea sativa & Luz & 19 \\
\hline 8 & Pseudotsuga menziesii - Acacia melanoxylon - Eucalyptus regnans & Sombra & 14 \\
\hline 9 & Pseudotsuga menziesii - Acacia melanoxylon - Eucalyptus regnans & Luz & 19 \\
\hline 10 & Quercus robur - Pseudotsuga menziesii - Acacia dealbata & Luz & 19 \\
\hline
\end{tabular}

Asociaciones 8 y 9 corresponden a nidos. Rodales 3 y 4 están compuestos por las mismas especies y se encuentran bajo la misma condición de luz, pero se ubican en diferentes sectores del predio.

Los casos 8 y 9 corresponden a nidos, pequeños grupos de 10 robles europeos distribuidos dentro de la plantación mixta. Se entiende como árboles potenciales $M \circ$ de madurez, aquellos árboles de selección (Schädelin, 1936 cit. por Siebert et al., 2003) que presumiblemente llegarán a la madurez y serán cosechados al alcanzar su diámetro objetivo (Siebert et al., 2003).

En términos prácticos, un árbol $M$ es dominante o codominante (estrato superior del bosque), recto (categoría 1), con una flecha (dominancia apical marcada) y sin daños (Cuadro $\mathrm{N}^{\circ}$ 2). Árboles que no cumplen con estas características son catalogados como no $M$. 


\begin{tabular}{|l|l|c|c|c|c|}
\hline Estrato & Rectitud & $\begin{array}{c}\text { Número } \\
\text { de } \\
\text { Flechas }\end{array}$ & Daño & $\begin{array}{c}\text { Tipo de } \\
\text { Arbol }\end{array}$ & Calidad \\
\hline $\begin{array}{l}\text { Dominante } \\
\text { Codominante }\end{array}$ & $1-$ Recto & 1 & $1-$ Sin & $M$ & Superior \\
$\begin{array}{l}\text { Dominante } \\
\text { Codominante } \\
\text { Intermedio } \\
\text { Suprimido }\end{array}$ & $\begin{array}{l}\text { 1- Recto } \\
\text { 2- Ligeramente Curvado } \\
\text { 3- Curvado en varias direcciones } \\
\text { 4- Fuertemente Curvado y/o torcido }\end{array}$ & 1 o más & $1-$ Sin & No $M$ & Inferior \\
\hline
\end{tabular}

En cuanto a la selección de los individuos, se eliminaron dos hileras de borde en cada parcela muestreada. El tamaño de la parcela se asignó proporcionalmente a la superficie del rodal, con un mínimo de 100 robles europeos por asociación.

En cada parcela se cuantificó todos los árboles $M$ y no $M$. Las variables (Cuadro $N^{\circ} 3$ ) cuantitativas medidas fueron DAP $(\mathrm{cm})$ y altura total $(\mathrm{m})$, así como la estratificación o posición sociológica, rectitud, bifurcación y daño según Wienstroer et al. (2003).

\section{Cuadro $\mathrm{N}^{\circ} 3$ \\ VARIABLES MEDIDAS EN EL ESTUDIO}

\begin{tabular}{|l|l|l|l|}
\hline \multicolumn{2}{|c|}{ Variables Cuantitativas } & \multicolumn{2}{c|}{ Variables Cualitativas } \\
\hline \multirow{2}{*}{ DAP } & $\begin{array}{l}\text { Medida del diámetro } \\
\text { fustal a 1,3 metros } \\
\text { desde el suelo }\end{array}$ & Estratificación & Dominante, Codominante, Intermedio y Suprimido. \\
\hline \multirow{2}{*}{ Altura Total } & $\begin{array}{l}\text { Altura desde la base } \\
\text { hasta el final de la } \\
\text { copa. }\end{array}$ & Rectitud & $\begin{array}{l}\text { Rectitud del fuste: 1) recto, 2) ligeramente curvado, 3) } \\
\text { curvado en varias direcciones, 4) fuertemente curvado } \\
\text { y/o torcido. }\end{array}$ \\
\cline { 3 - 4 } & & Bifurcación & Presencia o ausencia: 1) sin bifurcación, 2) bifurcado. \\
\cline { 2 - 4 } & Daño & Presencia o ausencia: 1) sin daño, 2) dañado. \\
\hline
\end{tabular}

La proporción de árboles de calidad a cosechar en la madurez $(M)$, se complementó con rectitud, incrementos en DAP y altura, de modo que la suma de factores determinó la bondad de la asociación.

\section{Análisis Estadísticos}

Se comparó la cantidad de robles europeos en cada asociación (rodales 2 a 10) y en plantación pura (rodal 1), considerando como variable dependiente (respuesta) su crecimiento (incremento en diámetro y altura), así como las variables presencia/ausencia de daño, bifurcación, rectitud y tipo de árbol, y como variable explicativa la asociación.

Dada la inexistencia de un diseño experimental, se utilizaron pseudo réplicas, que corresponden a subdivisiones aleatorias de la muestra que permiten analizar datos de esta naturaleza mediante modelos estadísticos lineales mixtos, que permiten analizar datos con estructuras de dependencia, desbalance y falta de normalidad (Balzarini et al., 2005). 
Para comparar diámetro y altura (variables respuesta de naturaleza continua) se utilizó un análisis de varianza para contrastar la hipótesis de igual crecimiento promedio de robles europeos en las distintas asociaciones.

Para las variables daño, bifurcación, tipo de árbol y rectitud, se utilizaron modelos para datos de naturaleza binaria, transformación logit que fue la que más se ajustó a la distribución de los datos.

En el caso de la rectitud, se transformaron las categorías no rectas (2, 3 y 4) para tratar la calidad fustal como variable binomial a través de la regresión logística. El modelo lineal clásico tiene la siguiente forma:

$$
y i j=\mu+t j+e i j
$$

Donde; $\quad y_{i j}$ es la variable dependiente (incremento en altura y/o diámetro)

$\mu$ la media generada de las variables dependientes

$t_{j}$ el efecto de la asociación

$e_{i j}$ el término del error aleatorio.

Por otra parte, el modelo de regresión logística:

$$
\text { Logit }(\pi)=B_{0}+\beta_{1}{ }^{*} A s o c
$$

Donde: $\pi$ proporción de éxito de la variable respuesta binaria en estudio

$B_{0}$ constante de la regresión logística

$B_{1}$ el coeficiente de la regresión logística

Asoc la asociación.

Para la proporción de árboles de calidad se utilizó el análisis de los Odds ratios o cocientes de chances, indicador que permite comparar éxito entre asociaciones respecto a un parámetro, en este caso la proporción de árboles futuro.

\section{RESULTADOS}

Las plantaciones mixtas se encontraban en su etapa inicial de desarrollo (13 a 20 años), en el primer tercio de la rotación estimada (60 años), por lo que el análisis y las posteriores conclusiones son válidos para este período, pudiendo sufrir variaciones a futuro.

La proporción de árboles $M$ fue mayor en la asociación $5(67,6 \%)$, seguido en orden decreciente por la $4(65,0 \%)$ y $10(60,3 \%)$ y la pura $(59,1 \%)$.

Los nidos (situaciones 8 y 9), se excluyeron de este análisis para ser estudiados separadamente por corresponder a una variante silvícola (Cuadro $\mathrm{N}^{\circ} 4$ ), presentando los valores menores $(40,0 \%$ y $17,0 \%$, respectivamente).

Dado que el manejo planificado eliminará cerca del $87 \%$ de los árboles (200 árboles finales por hectárea), la proporción de árboles $M$ aumentará durante la rotación. 
PORCENTAJE DE ÁRBOLES $M$ EN LAS ASOCIACIONES EVALUADAS

\begin{tabular}{|c|c|c|c|c|}
\hline $\begin{array}{c}\text { Rodal } \\
\text { № } \\
\end{array}$ & Asociación & Ladera & $\begin{array}{c}\text { Cantidad Robles } \\
\text { Europeos } \\
\left(\mathbf{N}^{\circ}\right) \\
\end{array}$ & $\begin{array}{c}\text { Proporción } \\
\text { Árboles } M \\
(\%)\end{array}$ \\
\hline 1 & Q. robur - Q. robur & Luz & 154 & 59 \\
\hline \multicolumn{3}{|c|}{ Subtotal Puro } & 154 & 59 \\
\hline 2 & Q. robur - C. sativa & Sombra & 152 & 51 \\
\hline 3 & \multirow{2}{*}{ Q. robur - A. pseudoplatanus - C. sativa } & Luz & 235 & 51 \\
\hline 4 & & Luz & 123 & 65 \\
\hline 5 & \multirow{2}{*}{ Q. robur - P. menziesii - C. sativa } & Luz & 145 & 68 \\
\hline 6 & & Sombra & 224 & 46 \\
\hline 7 & Q. robur - C. macrocarpa - C. sativa & Luz & 244 & 47 \\
\hline 8 & \multirow{2}{*}{ P. menziesii-A. melanoxylon - E. regnans } & Sombra & 97 & 40 \\
\hline 9 & & Luz & 128 & 17 \\
\hline 10 & Q. robur - P. menziesii - A. dealbata & Luz & 131 & 60 \\
\hline \multicolumn{3}{|c|}{ Subtotal Mixto } & 1.572 & 49 \\
\hline
\end{tabular}

Rodales 3 y 4 están compuestos por las mismas especies y se encuentran bajo la misma condición de luz, pero se ubican en diferentes sectores del predio.

El análisis de los Odds ratios en las asociaciones 5, 4, 10 y 1 (con valores más altos) indica una mayor proporción de árboles $M(68 \%)$ de la asociación 5 respecto a las demás. Las diferencias observadas entre la asociación 5 y las asociaciones 4 y 10 no fueron estadísticamente significativas (Cuadro $N^{\circ} 5$ ). La probabilidad de que las diferencias observadas hayan sido por azar fueron 0,61 y 0,72 , respectivamente.

Entre las asociaciones 1 y 5 , las diferencias fueron estadísticamente significativas $(p<0,08)$ con un Odd ratio de 0,64 , lo que indica que las chances de árboles $M$ en la asociación mixta 5 es 1,56 veces mayor que en la asociación pura. En sentido estricto, este valor p debió ser rechazado producto de que era mayor al admitido $(p<0,05)$, pero en este caso un valor $p<0,08$ se consideró aceptable dada la naturaleza del estudio, debido a que el valor $p$ depende de dos elementos esenciales, primero la magnitud de la diferencia a probar y segundo el tamaño de la muestra, que en este caso es reducido. Se debe tener en cuenta que si estos elementos no están adecuadamente considerados se generarán resultados que pueden finalmente llevar a una toma de decisiones incorrecta.

Cuadro $N^{\circ} 5$

VALORES DE COCIENTES DE CHANCE DE ÁRBOLES $M$ EN ASOCIACIONES 4, 10 Y 1 RESPECTO A LA ASOCIACIÓN 5, DE MAYOR PROPORCIÓN DE ÁRBOLES $M$

\begin{tabular}{|c|c|c|c|c|c|}
\hline \multicolumn{2}{|c|}{ 4s $\mathbf{5}$} & \multicolumn{2}{c|}{$\mathbf{1 0}$ vs $\mathbf{5}$} & \multicolumn{2}{c|}{$\mathbf{1}$ vs $\mathbf{5}$} \\
\hline Chance & Valor $\mathrm{p}$ & Chance & Valor $\mathrm{p}$ & Chance & Valor $\mathrm{p}$ \\
\hline 1,16 & 0,6138 & 1,11 & 0,7211 & 0,64 & 0,0801 \\
\hline
\end{tabular}


El análisis de asociaciones según laderas consideró el mismo criterio de análisis de cocientes de chances para la proporción de árboles $M$. En la asociación 5 , el análisis con un nivel de significación del $5 \%$ indica que la ladera soleada tiene $33 \%$ más chances de proporcionar árboles de calidad $M$ que la ladera sombría (Cuadro $N^{\circ} 6$ ).

\section{Cuadro $\mathrm{N}^{\circ} 6$}

VALORES DE COCIENTES DE CHANCE DE ÁRBOLES $M$ EN DISTINTA LADERA COMPARACIÓN DE ASOCIACIONES 6 - 5 Y 8 - 9

\begin{tabular}{|l|c|c|c|c|c|}
\hline \multirow{2}{*}{ Asociación } & \multirow{2}{*}{ Ladera } & \multicolumn{2}{|c|}{$\mathbf{6}$} & \multicolumn{2}{|c|}{$\mathbf{8}$} \\
\cline { 3 - 6 } & & Chance & $\mathbf{p}$ & Chance & \multicolumn{1}{|c|}{$\mathbf{p}$} \\
\hline Q. robur - P. menziesii - C. sativa & \multirow{3}{*}{ Soleada } & 0,33 & $<0,0001$ & & \\
\cline { 4 - 6 } P. menziesii - A. melanoxylon - E. regnans & & & 2,42 & 0,0077 \\
\hline
\end{tabular}

De igual forma, en los nidos ubicados en la asociación $P$. menziesii, $A$. melanoxylon y $E$. regnans ( 8 y 9 respectivamente), existe una mayor chance (2,42 veces superior) de tener árboles $M$ que su símil en ladera soleada $(p=0,0077)$.

Los mayores crecimientos en altura y diámetro, se encontraron en la situación pura (Cuadros $\mathrm{N}^{\circ} 7$ y $\mathrm{N}^{\circ} 8$ ).

\section{Cuadro $\mathrm{N}^{\circ} 7$}

CRECIMIENTO ANUAL EN DIÁMETRO A LA ALTURA DEL PECHO (DAP) PARA EL TOTAL DEL RODAL Y PARA ÁRBOLES $M$

\begin{tabular}{|c|c|c|}
\hline $\begin{array}{c}\text { Rodal } \\
\left(\mathrm{N}^{\circ}\right)\end{array}$ & $\begin{array}{l}\text { Incremento Medio DAP Árboles } M \\
\text { (cm/año) }\end{array}$ & $\begin{array}{l}\text { Incremento Medio DAP Todos los Árboles } \\
\text { (cm/año) }\end{array}$ \\
\hline 1 & $0,86 \quad \mathrm{a}$ & $0,80 \mathrm{~A}$ \\
\hline 4 & 0,74 & 0,68 \\
\hline 5 & 0,71 & 0,67 \\
\hline 8 & 0,68 & 0,59 \\
\hline 3 & 0,67 & 0,64 \\
\hline 6 & 0,62 & 0,59 \\
\hline 7 & 0,61 & $0,56 \mathrm{E}$ \\
\hline 2 & 0,60 & 0,56 \\
\hline 10 & 0,61 & 0,56 \\
\hline 9 & $0,49 \mathrm{f}$ & 0,39 \\
\hline
\end{tabular}

Letras distintas indican diferencias significativas $(p<0,05)$, según el Test LSD Fisher.

Asociaciones 8 y 9 corresponden a nidos.

\section{Cuadro $\mathrm{N}^{\circ} 8$ \\ CRECIMIENTO ANUAL EN ALTURA}

PARA EL TOTAL DEL RODAL Y PARA ÁRBOLES $M$

\begin{tabular}{|c|c|c|}
\hline $\begin{array}{c}\text { Rodal } \\
\mathbf{N}^{\circ}\end{array}$ & $\begin{array}{c}\text { Incremento Medio Altura Árboles } \boldsymbol{M} \\
\text { (m/año) }\end{array}$ & $\begin{array}{c}\text { Incremento Medio Altura Todos los Árboles } \\
\text { (m/año) }\end{array}$ \\
\hline 1 & $1,08 \mathrm{a}$ & $1,20 \mathrm{~A}$ \\
\hline 8 & $0,90 \mathrm{~b}$ & $0,77 \mathrm{cb}$ \\
5 & $0,81 \mathrm{c}$ & $0,80 \mathrm{~b}$ \\
3 & $0,81 \mathrm{dc}$ & $0,77 \mathrm{cb}$ \\
4 & $0,81 \mathrm{dc}$ & $0,77 \mathrm{cb}$ \\
7 & $0,80 \mathrm{dc}$ & $0,74 \mathrm{c}$ \\
10 & $0,77 \mathrm{ed}$ & $0,72 \mathrm{c}$ \\
2 & $0,75 \mathrm{e}$ & $0,72 \mathrm{c}$ \\
6 & $0,71 \mathrm{f}$ & $0,66 \mathrm{~d}$ \\
9 & $0,62 \mathrm{~g}$ & $0,50 \mathrm{e}$ \\
\hline
\end{tabular}

Letras distintas indican diferencias significativas $(p<0,05)$, según el Test LSD Fisher Asociaciones 8 y 9 corresponden a nidos. 
Tanto para el incremento anual en altura como en DAP existen diferencias significativas tanto para el total del rodal como para los árboles $M$. A nivel de rodal, el incremento diámetrico

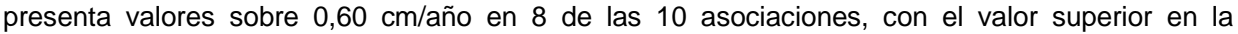
situación pura $(0,86 \mathrm{~cm} / \mathrm{año})$ y el inferior en la asociación $9(0,49 \mathrm{~cm} / a n ̃ o)$; el crecimiento anual en altura supera $0,80 \mathrm{~m} / \mathrm{año}$ en 5 asociaciones, con el valor superior en la situación pura $(1,08 \mathrm{~m} / \mathrm{año})$ y el inferior en la asociación 9 (0,62 m/año).

A nivel de árboles $M$, se registran valores en general inferiores, con crecimientos en

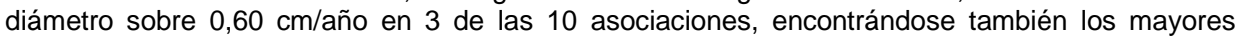
incrementos en la situación pura $(0,80 \mathrm{~cm} /$ año y 1,20 m/año respectivamente) y los inferiores en la asociación 9 para ambos parámetros $(0,39 \mathrm{~cm} /$ año y $0,50 \mathrm{~m} / \mathrm{año})$.

Los coeficientes de la regresión logística de la incidencia de daño, bifurcación, rectitud y tipo de árbol respecto a la asociación se presentan en el Cuadro $\mathrm{N}^{\circ} 9$.

\section{Cuadro $N^{\circ} 9$ \\ RESUMEN DE RESULTADOS PARA LOS MODELOS DE REGRESIÓN LOGÍSTICA}

\begin{tabular}{|l|c|c|r|}
\hline Variable & $\boldsymbol{B}_{0}$ & $\boldsymbol{B}_{1}$ & \multicolumn{1}{|c|}{ Valor $\mathbf{p}$} \\
\hline Daño & $-4,61$ & 0,17 & 0,0062 \\
Bifurcación & 1,85 & $-0,13$ & $<0,0001$ \\
Rectitud & 0,73 & $-0,02$ & 0,2944 \\
Tipo de árbol & 0,56 & $-0,06$ & 0,0021 \\
\hline
\end{tabular}

La asociación es un buen predictor tanto para bifurcación, que puede afectar fuertemente tanto la calidad como la cantidad de madera producida por un árbol (Hung et al., 2011), como para el tipo de árbol, existiendo diferencias significativas entre asociaciones.

Como se observa en el Cuadro $N^{\circ} 9$, los valores $\mathrm{p}$ son bajos (inferiores al $5 \%$ ) lo que implica un alto nivel de correlación entre la presencia de bifurcación y la formación de árboles de calidad. Por el contrario, la rectitud no presentó diferencias significativas $(p=0,2944)$, característica que no depende de la mezcla, sino que de aspectos genéticos u otros no estudiados.

Finalmente, los resultados de la proporción esperada para las distintas asociaciones respecto a árboles $M$, daño, bifurcación y rectitud se presentan en el Cuadro $N^{\circ} 10$. La síntesis final permite visualizar de forma integral las distintas variables y resultados del estudio (Cuadros $\mathrm{N}^{\circ} 11 \mathrm{y}$ $N^{\circ} 12$ ).

Cuadro $\mathrm{N}^{\circ} 10$

PROPORCIÓN ESPERADA DE ÁRBOLES SANOS, RECTOS, NO BIFURCADOS Y DE CALIDAD $M$

\begin{tabular}{|c|r|r|c|c|}
\hline \multirow{2}{*}{ Asociación } & Sano & Rectos & Sin Bifurcación & Árboles $\boldsymbol{M}$ \\
\cline { 2 - 5 } & \multicolumn{4}{|c|}{$\begin{array}{c}\text { Probabilidad } \\
\text { (\%) }\end{array}$} \\
\hline 1 (puro) & 99 & 65 & 90 & 63 \\
2 & 100 & 62 & 81 & 54 \\
3 & 96 & 66 & 75 & 58 \\
4 & 99 & 75 & 93 & 75 \\
5 & 99 & 79 & 88 & 73 \\
6 & 97 & 59 & 69 & 47 \\
7 & 100 & 65 & 64 & 49 \\
8 & 94 & 64 & 65 & 26 \\
9 & 87 & 39 & 58 & 71 \\
10 & 98 & 75 & 81 & \\
\hline
\end{tabular}


Cuadro $\mathrm{N}^{\circ} 11$

RESUMEN DE LOS RESULTADOS DEL ESTUDIO CLASIFICADOS POR ASOCIACIÓN

\begin{tabular}{|c|c|c|c|c|c|c|}
\hline \multirow[b]{2}{*}{ Asociación } & \multicolumn{2}{|c|}{ Incremento anual } & \multicolumn{4}{|c|}{ Proporción esperada de individuos (1 a 100\%) } \\
\hline & DAP & Altura & Rectos & $\begin{array}{c}\text { Sin } \\
\text { Bifurcación }\end{array}$ & Sin Daño & Árboles $M$ \\
\hline 1 (Puro) & 00001 & 00000 & 000 & ○९९०1 & 00000 & 00 \\
\hline 2 & $\bullet \bullet$ & $\bullet \bullet \bullet \bullet$ & $\bullet \bullet$ & 0000 & $\bullet \bullet \bullet \bullet \bullet$ & $\bullet \bullet$ \\
\hline 3 & 000 & 000 & 00 & 000 & 0 & 00 \\
\hline 4 & 000 & 00001 & 0000 & 00001 & 00000 & 000 \\
\hline 5 & ○००८ & 0000 & 0000 & ๑९००८ & ๑९९०० & ○०९ \\
\hline 6 & 000 & 0001 & 000 & 0001 & 00 & 001 \\
\hline 7 & $\bullet \bullet \bullet$ & 0000 & 00 & 000 & ००००० & 001 \\
\hline 8 & ○०० & ९०००८ & 000 & 000 & 00000 & 001 \\
\hline 9 & ○९ & 000 & 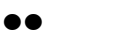 & $\bullet$ & ๑०००४ & 01 \\
\hline 10 & 000 & $\bullet \bullet \bullet \bullet$ & $\bullet \bullet \bullet \bullet$ & 000 & $\bullet \bullet \bullet \bullet \bullet$ & $\bullet 001$ \\
\hline
\end{tabular}

$=$ Valor equivalente a $0,2 \mathrm{~cm} \circ \mathrm{m}$, o $20 \%$ según el caso. Los valores aproximados a la figura, no representan necesariamente el valor exacto para la variable.

Cuadro $\mathrm{N}^{\circ} 12$

RESUMEN DE LOS RESULTADOS DEL ESTUDIO CATEGORIZADOS Y CLASIFICADOS POR ASOCIACIÓN

\begin{tabular}{|c|c|c|c|c|c|c|}
\hline \multirow[b]{2}{*}{ Asociación } & \multicolumn{2}{|c|}{ Incremento anual } & \multicolumn{4}{|c|}{ Proporción esperada de individuos } \\
\hline & DAP & Altura & Rectos & $\begin{array}{c}\text { Sin } \\
\text { Bifurcación }\end{array}$ & Sin Daño & Árboles $M$ \\
\hline 1 (Puro) & $\star \star \star \star$ & $\star \star \star$ & $\star \star$ & $\star \star \star \star$ & $\star \star \star \star$ & $\star \star$ \\
\hline 2 & $\star \star$ & $\star \star \star$ & $\star \star$ & $\star \star \star$ & $\star \star \star$ & $\star \star$ \\
\hline 3 & $\star \star \star \star$ & $\star \star \star$ & $\star \star$ & $\star \star$ & $\star \star \star \star$ & $\star \star$ \\
\hline 4 & $\star \star \star \star$ & $\star \star \star$ & $\star \star \star$ & $\star \star \star \star$ & $\star \star \star \star$ & $\star \star \star$ \\
\hline 5 & $\star \star \star \star$ & $\star \star \star \star$ & $\star \star \star \star$ & $\star \star \star \star$ & $\star \star \star \star$ & $\star \star \star \star$ \\
\hline 6 & $\star \star$ & $\star \star \star$ & $\star \star$ & $\star \star$ & $\star \star \star$ & $\star \star \star$ \\
\hline 7 & $\star \star$ & $\star \star \star$ & $\star \star$ & $\star \star$ & $\star \star \star \star$ & $\star \star$ \\
\hline 8 & $\star \star \star \star$ & $\star \star \star \star$ & $\star \star$ & $\star \star$ & $\star \star \star \star$ & $\star \star$ \\
\hline 9 & $\star \star$ & $\star \star$ & $\star$ & $\star \star$ & $\star \star \star$ & $\star \star$ \\
\hline 10 & $\star \star$ & $\star \star \star$ & $\star \star \star$ & $\star \star \star$ & $\star \star \star$ & $\star \star \star$ \\
\hline
\end{tabular}

Categorías: $\star \star \star=$ Excelente

$\star \star$ = Bueno

$\star=$ Regular

$=$ Malo

Las asociaciones 4, 5, 10 y 1 destacan por sobre el resto. Si bien los valores de las asociaciones 4 y 5 están catalogados como excelentes, el Cuadro $N^{\circ} 12$ muestra un mayor valor para la situación 4 en desmedro de la 5, por lo se puede afirmar que corresponde a la situación más favorable para el roble europeo, pues posee valores superiores en crecimiento y proporción de árboles $M$. 


\section{DISCUSIÓN}

La densidad de las plantaciones estudiadas (1.634 árboles/ha) es superior a las densidades de plantación empleadas en Italia, que oscilan entre 800 y 1.100 árboles/ha (Mercurio y Minotta, 2000). Para las variables estudiadas, se observaron diferencias entre asociaciones, en concordancia con otra experiencia en Chile reportada por Loewe y González (2009).

En relación a la proporción de árboles de calidad, las asociaciones 4, 5 y 10 son las más favorables para el desarrollo de árboles $M(65,68$ y $60 \%$, respectivamente), se encuentran en ladera soleada, y poseen una mezcla de especies que favorece la formación de individuos con aptitud para producir madera de calidad, siendo esto provechoso para el crecimiento inicial de $Q$. robur, la situación pura contiene un $59 \%$ de árboles $M$. Las especies acompañantes, además de minimizar la competencia por luz, proporcionan una protección lateral a la especie principal. De este modo, los fustes protegidos por el dosel del estrato intermedio producen maderas de mayor calidad, sin brotes epicórmicos.

Según Joyce et al. (1998), la asociación con coníferas generalmente cumple un rol protector, pues su hábito disminuye la penetración de luz provocando un desarrollo fustal con baja generación de brotes epicórmicos y un sistema radicular vigoroso.

Tanto en la asociación 5 como en la 10 está presente pino oregón como especie asociada, cuyo acompañamiento lateral favorece el desarrollo en altura de los individuos y la formación de ramas delgadas y poco vigorosas, pues su hábito reduce la penetración de luz al fuste. No obstante, este rol protector debe ser eliminado a tiempo, evitando la competencia con el roble europeo, y posteriormente continuar con ciclos de poda a 12-15 m de altura, siendo este el momento adecuado para cosechar algunas coníferas.

La asociación 10, junto a la protección fustal otorgada por el pino oregón, incluye regeneración natural de Acacia dealbata, cuya fijación de $\mathrm{N}$ podría explicar el buen comportamiento de esta asociación (Forrester et al., 2006).

En la asociación 4, la protección fustal es realizada por Acer pseudoplatanus y en menor medida por Castanea sativa, beneficiándose todas las especies del rodal. Siebert en González (2004), afirma que las plantaciones mixtas, no siempre coetáneas, utilizadas dentro de los límites de la sostenibilidad, mejoran el resultado económico, basado en una gestión silvícola a nivel de árbol individual (la biomasa no es el objetivo, aunque permite financiar las primeras intervenciones), con mayor valor por la calidad fustal (dimensión y calidad de la madera) de las especies arbóreas que se cosechan.

Se debe considerar que al ralear, con la mayor penetración de luz, el rodal reacciona elevando notoriamente los incrementos diamétricos y en altura; de hecho Buresti et al. (1998) señalan que en una plantación pura de roble europeo de 12 años, después del raleo, el incremento anual subió de 1 a 1,4 cm/año, junto a la aparición de numerosos brotes epicórmicos y aumento en grosor de las ramas, lo que provoca un deterioro en la calidad de la madera, aspectos que la excluyen de los exigentes mercados especializados europeos. Además, Loewe y González (2006) señalan que una plantación mixta de $Q$. robur con aliso italiano (Alnus cordata) tiene un crecimiento superior al cultivo mono específico, que es incluso 1 año mayor. Es importante señalar que el rápido crecimiento inicial del aliso italiano, de mayor desarrollo en altura y diámetro respecto al roble europeo, posteriormente decrece, con valores de incremento corriente inferiores a los del incremento medio.

Cabe señalar que la asociación de roble europeo con pino oregón, aromo australiano y Eucalyptus regnans (9) presentó el menor desarrollo, probablemente debido a la presencia simultánea de tres especies acompañantes vigorosas, que compiten por la luz y el espacio que el roble necesita para un buen desarrollo de su copa (Ravagni et al., 2015), y que presentan una tasa de crecimiento igual o mayor al de esta especie. 
Otra razón para no establecer la especie en plantaciones puras, es que en la plantación mixta la variabilidad de los incrementos es menor, simplificando el manejo (Loewe, 2002). Además, la asociación de especies reduce riesgos por elementos abióticos, como heladas, viento o sequías, que pueden dañar a una determinada especie, y no a otras; o debido a factores bióticos, ya que la presencia de varias especies en una plantación limita la propagación de enfermedades y ataques parasitarios. Además, disminuye la incertidumbre económica por variaciones de los mercados y por la duración de los ciclos de cultivo (Loewe y González, 2001).

Una de las características más notables de las plantaciones mixtas es su flexibilidad, generando un mayor margen para cosechar en momentos económicamente atractivos, lo que representa beneficios al comercializar el producto. Las especies acompañantes pueden inducir uno o más efectos positivos en la asociación, estimulando el crecimiento de la especie principal, favoreciendo una dominancia apical elevada y la formación de ramas delgadas. Además, inducen un notable desarrollo en altura los primeros años y un sucesivo ritmo de crecimiento menor, que proporciona un estímulo inicial en el crecimiento de la especie principal y sucesivamente ejerce una menor competencia por luz y espacio.

En general, el disponer de especies secundarias capaces de producir bienes complementarios en el transcurso de la rotación es importante, ya que el ingreso principal de una plantación es lejano en el tiempo respecto al momento de la plantación, siendo este un factor a considerar para el mercado chileno.

El efecto de los nidos de roble europeo en la proporción de árboles $M$ fue pobre $(40 \%$ en ladera sombría y $17 \%$ en ladera soleada). No obstante, la incorporación de grupos reducidos dentro de plantaciones proporcionará unos 15 robles europeos de máxima calidad por hectárea, con diámetros sobre $60 \mathrm{~cm}$ sin nudos, anillos de crecimiento uniformes y de color claro, características que se forman por la tenue inclusión de luz y un crecimiento pausado, altamente valorados en el mercado. No obstante, se sugiere establecer nidos en otras asociaciones a fin de evaluar si es un comportamiento generalizado para esta variante silvicultural.

Contrariamente a lo indicado por varios autores (Kerr, 2004; Gabriel et al., 2005; Gauthier et al., 2013), la plantación pura presentó mayor crecimiento medio que las mixtas tanto en altura como en diámetro $(0,80 \mathrm{~cm} / \mathrm{año}$ versus $0,58 \mathrm{~cm} / \mathrm{año})$, con un incremento diámetrico similar al inferior reportado por Ravagni et al. (2015) en plantación pura en Italia central. Sin embargo, esta modalidad de plantación no resulta adecuada para la producción de madera de calidad de la especie, pues con el manejo tradicional se induce el desarrollo de características no deseables para la madera de alto valor (brotes epicórmicos, individuos tipo "escobilla de mamadera", engrosamiento de ramas, etc.), factores que reducirán su precio.

\section{CONCLUSIONES}

Las plantaciones mixtas tienen ventajas para varios parámetros de importancia económica para el cultivo del roble europeo, con efectos positivos en su calidad fustal. Las asociaciones más favorables para la especie incluyen arce y castaño, seguido de pino oregón y castaño, y pino oregón y aromo, y luego por la plantación pura, en orden decreciente.

Varias asociaciones $(3,2,6$ y 7$)$ son propicias para el desarrollo de árboles $M$, siendo el manejo el encargado de mejorar su desarrollo. La plantación en grupos o nidos, presentó resultados pobres, comportándose mejor en exposición sombría.

El incremento en diámetro y altura fue superior en la situación pura, pero se estimó que en esta modalidad de plantación la proporción de árboles no rectos sobrepasaría el $30 \%$. Las mejores asociaciones poseen un crecimiento diamétrico elevado, al igual que la proporción de árboles rectos, debido a la presencia de especies acompañantes que favorecen un buen desarrollo de los individuos, primordial para formar fustes de calidad. 
Los resultados evidencian que ciertas especies acompañantes pueden desempeñar un rol importante en el cultivo del roble europeo, incidiendo en factores que determinan la calidad de la madera, como la rectitud y la presencia de bifurcaciones. Adicionalmente, también ejercerían un rol protector ante plagas y enfermedades, lo que en este caso no se detectó dado el buen estado sanitario de las plantaciones estudiadas.

\section{REFERENCIAS}

Aguayo, H., 2000. Evaluación del crecimiento en una plantación de Quercus robur L. en el sector de la región de la Araucanía. Tesis Ingeniero Forestal. Concepción, Chile. Facultad de Ciencias Forestales, Universidad de Concepción. p. $2-16$.

Álvarez, P.; Barrio, M.; Díaz, R.; Higueras, J.; Riesco, G.; Rigueiro, A.; Rodríguez, R. y, Villarino, J., 2000. Manual de selvicultura de frondosas caducifolias. Universidad Santiago de Compostela.

Balzarini, M.; Macchiavelli, R. y Casanoves, F., 2005. Aplicaciones de modelos mixtos en agricultura y forestería. Notas de clase Curso Internacional Aplicaciones de Modelos Mixtos en Agricultura y Forestería. CATIE. Turrialba, Costa Rica, 189p.

Berti, S.; Brunetti, M. y Rescic, L., 2003. Manuale sulla valutazione della qualità degli assortimenti legnosi ritraibili dalle specie legnose pregiate. Regione Lombardia. CNR-IVALSA, ARSAF. 48 p.

Burschel, P. y Huss, J., 1997. Grundriss des Waldbaus Ein Leitfaden für Studium and Praxis. Berlín, Alemania. Parey Buchverlag. p. $386-395$

Buresti, E.; De Meo, F. y Pelleri, F. ,1998. Criteri e risultati di un diradamento in un impianto di arboricultura da legno di farnia. Arezzo, Italia. Annali Istituto Sperimentale Selvicoltura - Arezzo. p. 29 - 40.

Ducousso, A. y Bordacs, S., 2004. EUFORGEN Technical guidelines for genetic conservation and use for pedunculate and sessile Oaks (Quercus robur and Quercus petraea). Roma, Italia. Plant Genetic Resources Institute. $6 \mathrm{p}$.

Forrester, D. I.; Bauhus, J.; Cowie, A. L. y Vanclay J. K., 2006. Mixed-species plantations of Eucalypts with nitrogen fixing trees: A review. Forest Ecology and Management 233 (2006) 211-230.

Forrester, D. I.; Vanclay, J. K. y Forrester, R. I., 2011. The balance between facilitation and competition in mixtures of Eucalypts and Acacia changes as stand develop. Oecologia 166 (2011) 265-272.

Gabriel, K., Blair, I. F. y Mason W. I., 2005. Growing broadleaves trees on the North York Moors, results after nearly 50 years. Quarterly Journal of Forestry 99 (2005), pp. 21-30.

Gauthier, M. M.; Zellers, K. E.; Löf, M. y Jacobs, D. F., 2013. Inter- and intra-specific competitiveness of plantation-grown American Chestnut (Castanea dentata).

González, Y., 2004. Efecto de diferentes asociaciones de especies vegetales en el crecimiento y la calidad fustal de aromo australiano o Blackwood (Acacia melanoxylon $\mathrm{R}$. Br.), Fundo Miraflores, Comuna de Lanco, X región, Chile. Tesis Ingeniero Forestal. Temuco, Chile, Facultad de Ciencias Forestales, Universidad de Temuco. p. 18 55 .

Hung, T. D.; Herbohn, J. L.; Lamb, D. y Nhan, H. D., 2011. Growth and production varies between pair-wise mixtures and monoculture plantations in North Viet Nam. Forest Ecology and Management 262 (2011) 440-448.

Joyce, P.; Huss, J.; Mc Carthy, R.; Pfeifer, A. y Hendrick, E., 1998. Growing Broadleaves, Silvicultural guidelines for Ash, Sycamore, Wild Cherry, Beech and Oak in Ireland. Dublin, Irlanda, National University of Ireland. COFORD. pp. $97-120$.

Kerr, G., 2004. The growth and form of Ash (Fraxinus excelsior) in mixture with Cherry (Prunus avium), Oak (Quercus petraea and Quercus robur), and Beech (Fagus sylvatica). Canadian Journal of Forest Research 34:2340-2350 
Loewe, V., 1998. Antecedentes de mercado de especies promisorias para Chile (aliso común, aliso rojo, arce, avellano, castaño, cerezo, encino rojo, fresno, grevillea, liquidambar, nogal común, nogal negro, pino piñonero, tulipero). Valdivia, Chile, INFOR. 132 p. Documento de Trabajo № 599.

Loewe, V., 2000. Recomendaciones para el cultivo de latifoliadas de alto valor. Santiago, Chile, INFOR - FIA. 40 p.

Loewe, V., 2002. Técnicas de poda para producir maderas duras de alta calidad y valor. Santiago, Chile, INFOR -FIA. 59 p.

Loewe, V., 2003. Arboricultura para producción de madera de alto valor. Santiago, Chile, INFOR - FIA. 56 p.

Loewe, V., 2009. Usos y Mercados de la Madera de Encino o Roble Europeo. Chile Forestal 345:45-49.

Loewe, V. y González, M., 2001. Plantaciones mixtas, una técnica de innovación productiva apropiada para la producción de maderas valiosas. Santiago, Chile, INFOR - FIA. 32 p.

Loewe, V. y González, M., 2006. Plantaciones Mixtas, Un modelo productivo con potencial para Chile. INFORFIA, $299 \mathrm{p}$.

Loewe, V. y González, M., 2009. Evaluación del crecimiento de Roble rojo americano (Quercus rubra), Encino (Quercus robur), Cerezo (Prunus avium) y Castaño (Castanea sativa) en plantaciones mixtas y pura orientadas a la producción de madera de alto valor en el sur de Chile en un periodo de siete años. XIII Congreso Forestal Mundial 2009, Buenos Aires, Argentina, 18-25 Octubre 2009.

Loewe, V.; González, M. y Balzarini, M. 2013. Wild Cherry tree (Prunus avium) growth in pure and mixed plantations of South America. Forest Ecology and Management Pp 31-41. Doi10.1016/j.foreco.2013.06.015.

Menéndez, J., 2006. Quercus robur L. Asturnatura.com [en línea], Num 97, 19/10/2006. Consultado el 10/09/2008. Disponible en: http://www.asturnatura.com/especie/quercus-robur.html

Mercurio, R. y Minotta, G., 2000. Arboricoltura da legno. Ed. CLUEB. Bolonia, Italia. 203 p.

Mori, P.; Bruschini, S.; Buresti Lattes, E.; Giulietti, V.; Grifoni, F.; Pelleri, F.; Ravagni, S.; Berti, S. Y Crivellaro, A., 2007. La selvicoltura delle specie sporadiche in Toscana. ARSIA, Florencia, Italia. 180 p.

Najera, Y.; Angulo, F. y López, V., 1969. Estudio de las principales maderas comerciales de frondosas peninsulares. Madrid, España, Instituto Forestal de Investigación y Experiencias. pp. $211-217$.

Piotto, D.; Víquez, E.; Montagnini, F. y Kanninen, M., 2004. Pure and mixed forest plantations with native species of the dry tropics of Costa Rica: a comparison of growth and productivity. Forest Ecology and Management 190: 359-372.

Ravagni, S.; Bidini, C.; Bianchetto, E.; Vitone, A. y Oelleri, F., 2015. Cultivation techniques in a 34 years old farming Oak (Quercus robur L.) plantation in the Arno valley (Tuscany). Annals of Silvicultural Research 39 (2) $111-117$.

Rodríguez, M., 1990. Geografía agrícola de Chile. 2ª Edición. Santiago, Chile, Universitaria. Pp 238 - 241.

Siebert, H.; Von Einsiedel, S. y Freiin Truchsess, A., 2003. Mejoramiento de la calidad fustal en plantaciones de Pseudotsuga menziesii al crecer en asociación con Acacia melanoxylon. Bosque 24 (3): 75 - 83 p.

Savill, P., 1991. The Silviculture of trees used in British Forestry. Oxford, Reino Unido, CAB. 179 p.

Suszka, B.; Muller, C. y Bonnet-Massimbert, M., 1994. Graines des feuillus forestiers de la récolte au semis. Paris, Francia, INRA. p. $247-249$.

Wienstroer, M.; Siebert, H. y Müller-Using, B., 2003. Competencia entre tres especies de Nothofagus y Pseudotsuga menziesii en plantaciones mixtas jóvenes, establecidas en la precordillera de Valdivia. Bosque 24 (3). $17-30 \mathrm{p}$. 